04.2

\title{
Влияние градиента концентрации плазмы на возбуждение ионно-циклотронных колебаний в омических разрядах токамака ТУМАН-3М
}

\author{
() А.А. Белокуров, Г.И. Абдуллина, Л.Г. Аскинази, Н.А. Жубр, В.А. Корнев, С.В. Крикунов, С.В. Лебедев, \\ Д.В. Разуменко, А.С. Тукачинский
}

Физико-технический институт им. А.Ф. Иоффе РАН, Санкт-Петербург, Россия

E-mail: belokurov@mail.ioffe.ru

Поступило в Редакцию 5 июня 2019г.

В окончательной редакции 5 июня 2019г.

Принято к публикации 10 июня 2019г.

В токамаке ТУМАН-ЗМ в разрядах с омическим нагревом обнаружены колебания на ионно-циклотронной (ИЦ) частоте в разрядах в дейтерии и водороде. Быстрые магнитные зонды, расположенные по полоидальному обходу плазменного шнура, способны различить несколько гармоник ионно-циклотронной частоты основного изотопа плазмы. При инжекции топливной макрочастицы (или пеллеты) интенсивность омических ИЦ-колебаний существенно уменьшается, а после полного испарения пеллеты возвращается к исходному уровню. Определение локализации и условий возбуждения ИЦ-колебаний в омическом режиме представляет особый интерес. На основе теории возбуждения дрейфово-циклотронной неустойчивости и численного моделирования сценариев с омическим переходом в режим улучшенного удержания и с инжекцией топливной пеллеты исследовано влияние параметров плазмы, в первую очередь градиента концентрации, на возможность возбуждения ИЦ-колебаний.

Ключевые слова: плазма, токамак, ионно-циклотронные колебания, пеллет-инжекция.

DOI: 10.21883/PJTF.2019.18.48234.17907

В плазме токамака ТУМАН-3М были обнаружены колебания на ионно-циклотронной частоте в омических разрядах и разрядах с нейтральным нагревным пучком. Наблюдение магнитных колебаний на токамаке ТУМАН-3М производится с помощью массива из шестнадцати быстрых магнитных зондов, расположенных по полоидальному обходу внутри вакуумной камеры. Полоса пропускания усилителей и частота преобразования АЦП позволяют зарегистрировать колебания частотой вплоть до $100-120 \mathrm{MHz}$. Более подробное описание диагностики и технические характеристики зондов приведены в [1].

В эксперименте стабильно наблюдается несколько гармоник омических ионно-циклотронных (ИЦ) колебаний на частоте основного изотопа плазмы. Пространственная и временна́я структура, а также причины специфического соотношения между интенсивностью различных гармоник омических ИЦ-колебаний являются предметом для отдельного исследования и не будут освещены в настоящей работе. Основная цель данного исследования заключается в том, чтобы выяснить степень влияния параметров плазмы, в первую очередь электронной концентрации и градиента концентрации, на возможность возбуждения ИЦ-колебаний.

В сценариях с инжекцией замороженной топливной макрочастицы (пеллеты) из-за испарения пеллеты происходит значительное изменение профиля концентрации в периферийной области плазмы. Рост периферийной концентрации также обусловлен тем, что испарение пеллеты инициировало переход в режим улучшенного удержания (Н-моду) [1]. Н-мода существует в течение примерно $3 \mathrm{~ms}$, затем происходит обратный переход в L-моду. Описанная эволюция режимов удержания плазмы видна на сигнале среднехордовой концентрации плазмы и уровня свечения линии $\mathrm{D}_{\alpha}$ (рис. $1, a$ ); момент перехода в Н-моду заметен на сигнале быстрых магнитных зондов: интенсивность омических ИЦ-колебаний (ohmic ICE) после испарения пеллеты и перехода в Нмоду существенно уменьшается (начиная с четвертой гармоники исчезает полностью), затем после обратного перехода в L-моду ИЦ-колебания регистрируются вновь с интенсивностью, превышающей значения до испарения пеллеты (рис. 1,a).

В характерных омических разрядах со спонтанным переходом в режим улучшенного удержания активность ИЦ-колебаний проявляется на финальной стадии разряда (рис. 2,a). Если в разряде присутствовал переход в H-моду, то примерно через $10 \mathrm{~ms}$ после LH-перехода магнитные зонды регистрируют несколько гармоник ИЦ-колебаний. В некоторых аналогичных разрядах без LH-перехода ИЦ-колебаний зарегистрировано не было или же колебания слабо выделялись на фоне шума (рис. 3, a). Таким образом, можно предположить, что наличие увеличенного градиента концентрации, появляющегося при переходе в Н-моду, способствует возбуждению ИЦ-колебаний.

Для того чтобы оценить степень влияния градиента концентрации на возможность возбуждения 
ИЦ-колебаний, была привлечена теория развития дрейфово-циклотронной неустойчивости [2,3]. Согласно этой теории, в неоднородной плазме колебания с частотами, кратными ионно-циклотронной, могут быть неустойчивыми при длине волны, меньшей ионного ларморовского радиуса $\rho_{i}$. При поперечном распространении волны условие возникновения неустойчивости выглядит следующим образом [3]:

$$
\chi \rho_{i}>2 \sqrt{\frac{m_{e}}{m_{i}}} \sqrt{1+\left(\frac{\omega_{c e}}{\omega_{p e}}\right)^{2}}
$$

или

$$
\chi \rho_{i}-2 \sqrt{\frac{m_{e}}{m_{i}}+\left(\frac{V_{\mathrm{A}}}{c}\right)^{2}}>0 .
$$

Здесь $\chi=\left|\frac{1}{n} \frac{\partial n}{\partial r}\right|$ - обратная характерная длина изменения концентрации плазмы, $\omega_{c e}$ и $\omega_{p e}-$ циклотронная и плазменная частоты электронов соответственно, $V_{\mathrm{A}}-$ альфвеновская скорость, $c-$ скорость света, $m_{e}$ и $m_{i}-$ массы электронов и ионов соответственно. В дальнейшем величину разности в формуле (1), описывающую выполнение критерия, будем обозначать как $\Delta K$; положительные значения $\Delta K$ соответствуют выполнению условий возбуждения ИЦ-колебаний.

В предположении, что омические ИЦ-колебания в токамаке ТУМАН-3М распространяются поперечно, данный критерий был применен к характерным экспериментальным сценариям с пеллет-инжекцией и омическим LH-переходом.

На основе показаний интерферометрической диагностики и периферийных измерений концентрации плазмы ленгмюровским зондом для исследуемых сценариев восстанавливался профиль концентрации плазмы; профиль ионной температуры был построен при помощи численного моделирования посредством кода ASTRA [4] и нормировки на измеренную с помощью корпускулярной диагностики температуру ионов в центре плазменного шнура. Влияние испарения пеллеты на профили концентрации и температуры было рассчитано с помощью кода ASTRA.

Зная распределение параметров плазмы, можно проверить выполнение условий критерия (1) при эволюции разряда. В различные моменты времени соотношение может меняться: как видно из формулы (1), более активной раскачке ИЦ-колебаний способствует больший относительный градиент концентрации, бо́льшая ионная температура и меньшая величина магнитного поля.

Рассмотрим сценарий с пеллет-инжекцией (рис. 1,a). Профиль концентрации до пеллет-инжекции соответствует выполнению критерия (1) вблизи от места расположения регистрирующего ИЦ-колебания магнитного зонда HFS (на стороне сильного магнитного поля, см. рис. $1, b)$. Моделирование эволюции концентрации и температуры плазмы при испарении пеллеты показало, что в области глубже транспортного барьера происходит уплощение профиля концентрации, что приводит к прекращению выполнения критерия (1) в этой области. Такое предположение соответствует экспериментально наблюдаемому уменьшению интенсивности омических ИЦ-колебаний. Тем не менее на нижних гармониках колебания в эксперименте продолжают регистрироваться быстрым магнитным зондом. Возможно, это связано с тем, что критерий (1) продолжает выполняться в других пространственных областях разряда. После испарения пеллеты и выхода из Н-моды градиент концентрации превышает значение до инжекции, что соответствует лучшему выполнению критерия (1) в более широкой области (рис. $1, b)$. Такое поведение качественно совпадает с экспериментальными наблюдениями: после испарения пеллеты интенсивность ИЦ-колебаний превышает первоначальный уровень. Необходимо отметить, что в данном разряде после $70 \mathrm{~ms}$ газонапуск был существенно менее интенсивным, чем в районе $50 \mathrm{~ms}$. Возможно, меньшее охлаждение периферии, обусловленное меньшим газонапуском, также способствовало увеличению интенсивности омических ИЦ-колебаний (так как ионная температура входит в $\chi \rho_{i}$, левую часть критерия (1)).

Для пары характерных омических разрядов (рис. 2, $a$, $3, a)$, различающихся наличием и отсутствием LH-перехода, был проведен схожий анализ выполнения критерия (1). На основе данных интерферометрической диагностики был построен профиль электронной концентрации; также параметры периферийной плазмы (концентрация, электронная температура) определялись при помощи ленгмюровского зонда. На основе данных профилей концентрации оказалось возможным проверить выполнение критерия (1) в разрядах с омическим LH-переходом и без LH-перехода. Результаты представлены на рис. $2, b$ и $3, b$.

Из рис. 2, $b$ (разряд с омическим LH-переходом) видно, что для начального профиля концентрации (50 ms) критерий не выполняется как на стороне слабого (LFS), так и на стороне сильного поля (HFS). Такой результат соответствует отсутствующим или неразличимым на уровне фона ИЦ-колебаниям в начальной фазе разряда (рис. 2,a). При переходе в Н-моду градиент на периферии увеличивается, в то же время ближе к концу разряда (80 ms) магнитное поле спадает, эти факторы способствуют возбуждению ИЦ-колебаний в периферийной области разряда (рис. 2,a). В конце разряда увеличенный градиент концентрации сохраняется, так как плазма остается в режиме улучшенного удержания. Радиальный профиль величины $\Delta K$ (локальное выполнение критерия (1)) показан на рис. 2, $b$.

В сценарии с LH-переходом критерий (1) выполняется на стороне слабого поля с середины малого радиуса до последней замкнутой магнитной поверхности и в узкой (примерно $1 \mathrm{~cm}$ ) области снаружи от нее (рис. 2, b). Концентрация плазмы на периферии, измеренная с помощью ленгмюровского зонда, хорошо „сшивается“ с профилем концентрации; таким образом, на основе зондовых измерений можно заключить, что в области разомкнутых силовых линий критерий (1) может выполняться только 

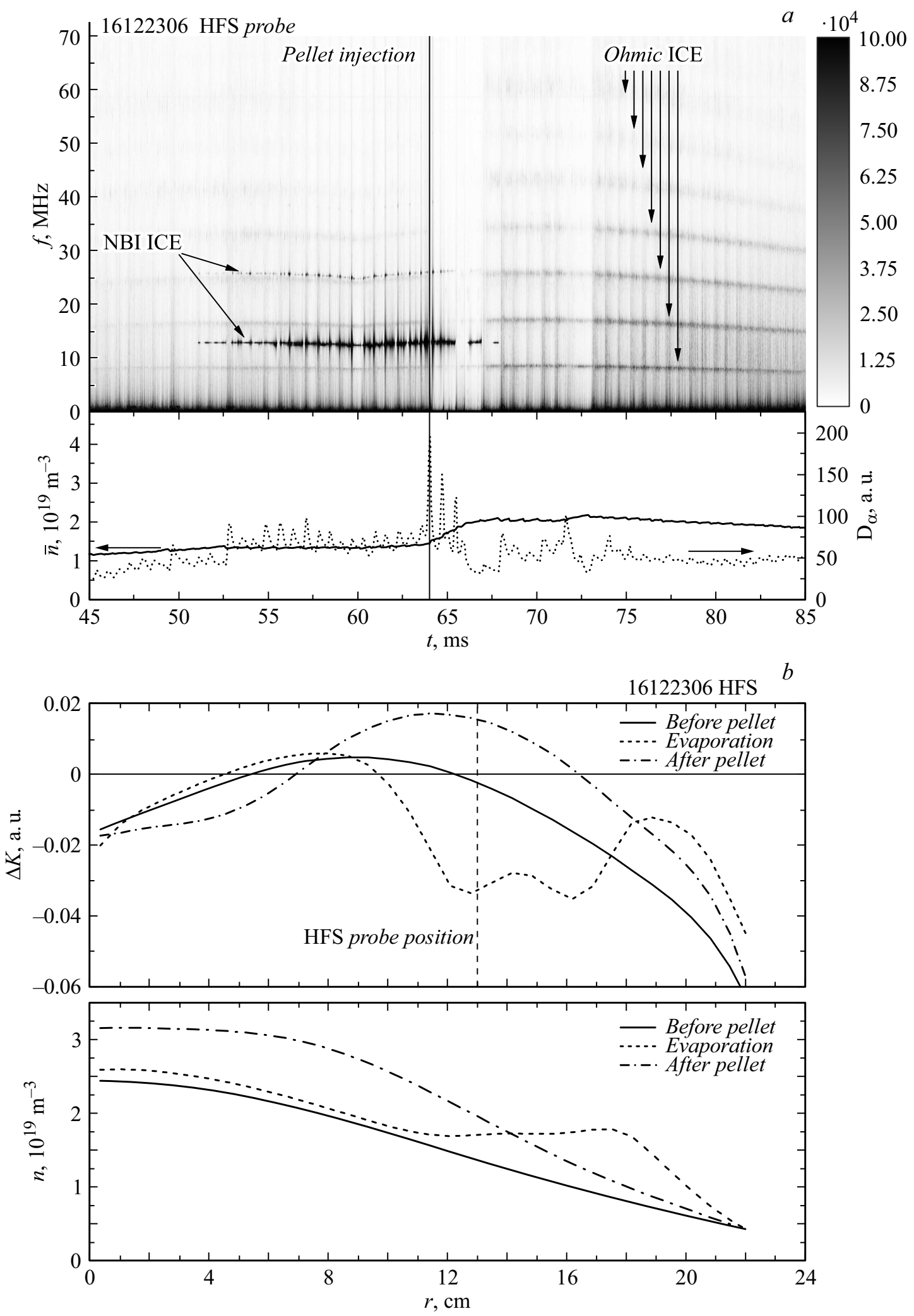

Рис. 1. $a-$ спектрограмма магнитных колебаний и эволюция среднехордовой электронной концентрации и свечения линии $\mathrm{D}_{\alpha}$ в разряде с пеллет-инжекцией $(64 \mathrm{~ms})$ и вызванным испарением пеллеты LH-переходом. NBI ICE - ионно-циклотронные колебания, вызванные быстрыми ионами при инжекции нейтрального нагревного пучка. $b$ - расчетная эволюция профиля электронной концентрации при пеллет-инжекции и локальное выполнение критерия (1) $(\Delta K>0)$ в зависимости от параметров плазмы в три момента времени: до инжекции, в максимуме кривой испарения и после испарения. 

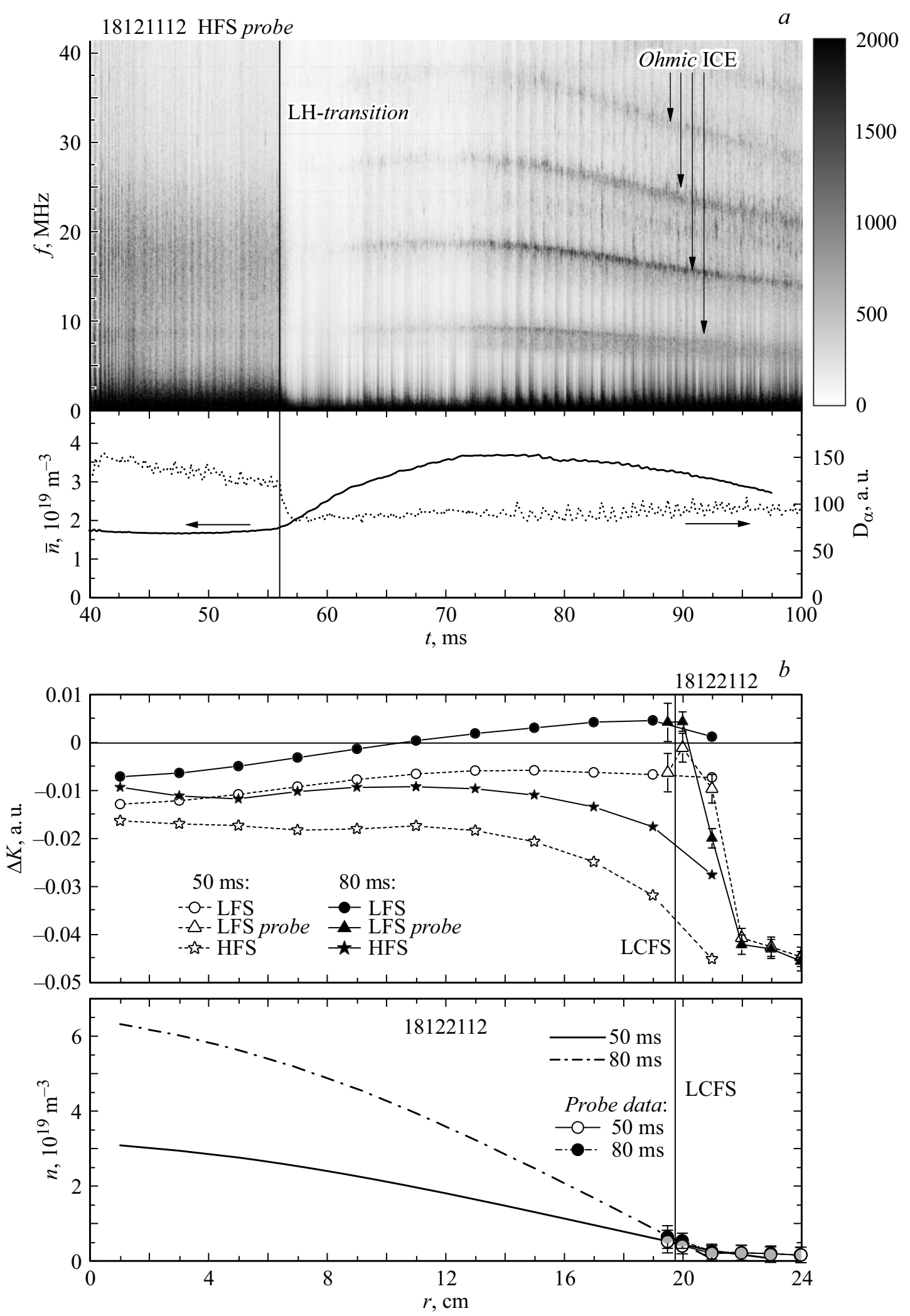

Рис. 2. $a$ - спектрограмма магнитных колебаний и эволюция среднехордовой электронной концентрации и свечения линии $\mathrm{D}_{\alpha}$ в разряде с омическим LH-переходом (на $\left.56 \mathrm{~ms}\right) . b-$ выполнение критерия (1) $(\Delta K>0)$ в зависимости от радиуса и соответствующие профили электронной концентрации плазмы в начальной фазе разряда $(50 \mathrm{~ms})$ и в фазе улучшенного удержания $(80 \mathrm{~ms})$. После LH-перехода градиент концентрации увеличивается, что приводит к возбуждению ИЦ-колебаний. Вертикальная линия LCFS показывает положение последней замкнутой магнитной поверхности. 

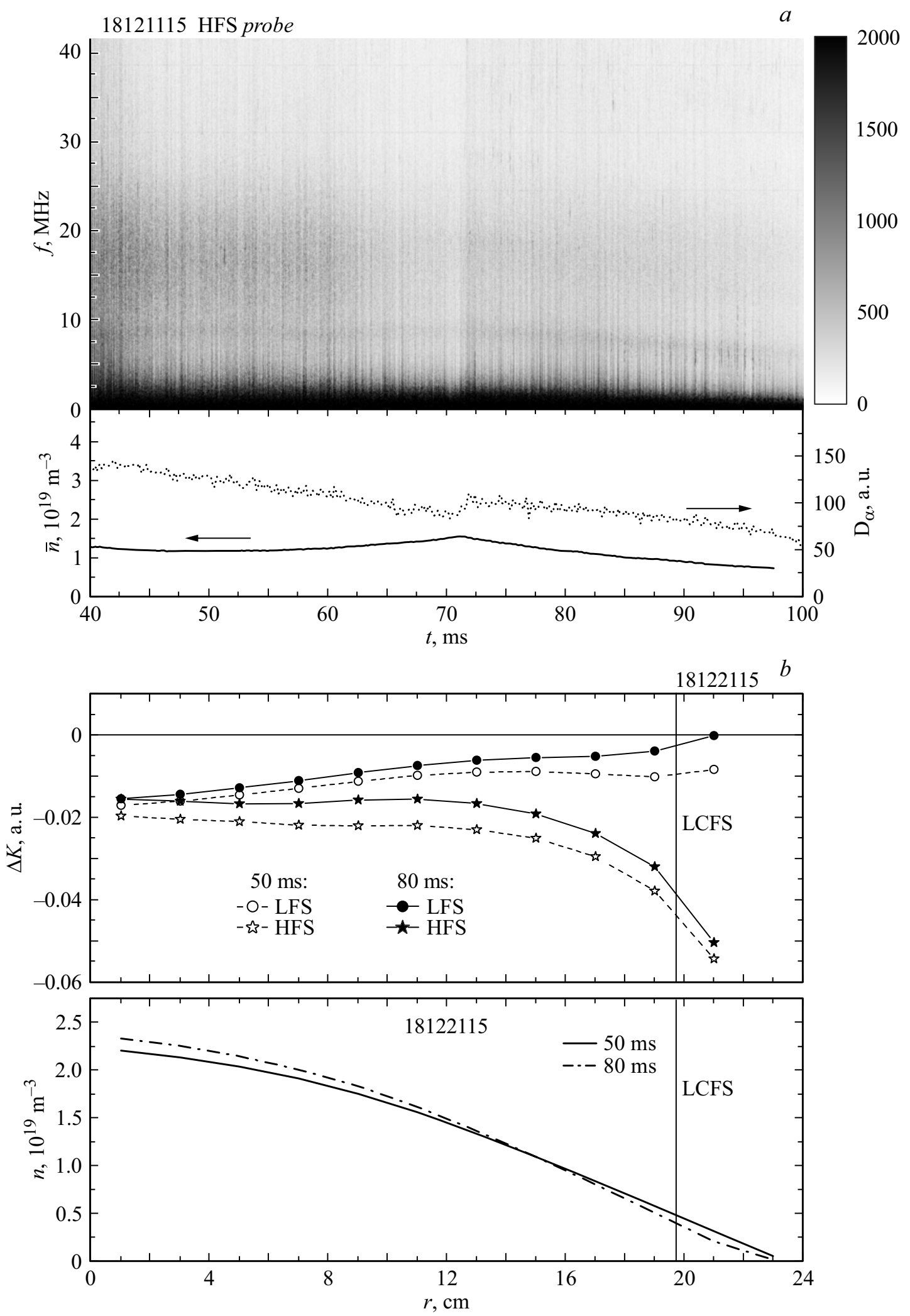

Рис. 3. $a-$ спектрограмма магнитных колебаний и эволюция среднехордовой электронной концентрации и свечения линии $\mathrm{D}_{\alpha}$ в разряде без улучшения удержания. $b-$ выполнение критерия (1) $(\Delta K>0)$ в зависимости от радиуса в два момента времени $(50$ и $80 \mathrm{~ms})$ и соответствующие профили концентрации плазмы. Профиль концентрации меняется незначительно, поэтому явного возбуждения ИЦ-колебаний не происходит. Вертикальная линия LCFS показывает положение последней замкнутой магнитной поверхности. 
рядом с последней замкнутой магнитной поверхностью, и ИЦ-колебания, согласно теории, с наибольшей вероятностью должны возбуждаться в основном объеме плазмы на периферии или же в области примерно $1 \mathrm{~cm}$ шириной снаружи от последней замкнутой магнитной поверхности.

Необходимо указать на факт, не укладывающийся в концепцию, определяющую возбуждение ИЦ-колебаний согласно критерию (1). Согласно расчетам, на стороне сильного поля не должно происходить возбуждения неустойчивостей, приводящих к ИЦ-колебаниям (см. линии для HFS на рис. 2, $b$ ), в то время как экспериментально более выраженный сигнал наблюдается именно на стороне сильного поля. Частота наблюдаемых ИЦ-колебаний соответствует значениям большого радиуса, близким к координатам магнитных зондов на стороне сильного поля, что свидетельствует в пользу того, что ИЦ-колебания возбуждаются также на стороне сильного магнитного поля.

На рис. 3, $b$ представлены результаты расчетов выполнения критерия (1) в омическом разряде без перехода в режим улучшенного удержания. Изменения профиля концентрации плазмы несущественно влияют на возможность возбуждения ИЦ-колебаний, так как по всему малому радиусу критерий (1) не выполняется. Экспериментальные наблюдения показывают слабые (соотношение сигнал-шум примерно 1.2) колебания на ИЦ-частоте. Учитывая, что профиль электронной концентрации восстановлен по хордовым сигналам с некоторой погрешностью, можно допускать генерацию ИЦ-колебаний на периферии со стороны слабого поля (где критерий (1) ближе всего к выполнению). Тем не менее такие ИЦ-колебания оказываются существенно слабее, чем в разряде с LH-переходом (где соотношение сигнал-шум на той же фундаментальной гармонике примерно равно 4.5).

На основании анализа разрядов с эволюцией ИЦ-колебаний, в которых существенно меняется градиент концентрации, можно сделать вывод о существенном влиянии формы профиля концентрации плазмы на возбуждение ИЦ-колебаний: больший градиент способствует раскачке ИЦ-колебаний, в то время как уплощение профиля, наоборот, приводит к тому, что ИЦ-колебания имеют меньшую интенсивность.

Тем не менее анализ на основе расчета выполнения критерия (1) не дает однозначного ответа на вопрос о действительной локализации колебаний. Несмотря на установленную качественную зависимость возможности генерации ИЦ-колебаний от градиента концентрации, совпадающую с экспериментальными наблюдениями, некоторые наблюдения оказывается невозможно описать с помощью предложенной в работах $[3,4]$ модели. Возможная причина расхождения заключается в недостаточно точно определенных профилях концентрации (особенно в области транспортного барьера) и ионной температуры.
Наблюдаемая в эксперименте асимметрия (более интенсивные колебания на стороне сильного поля и выше экваториальной плоскости), а также временна́я и спектральная структура являются предметом отдельного исследования.

\section{Финансирование работы}

Работа выполнена при поддержке Физико-технического института им. А.Ф. Иоффе (проведение экспериментов на токамаке ТУМАН-3М) и Российского научного фонда (моделирование и анализ разрядов с ИЦ-колебаниями, проект № 16-12-10285-П).

\section{Конфликт интересов}

Авторы заявляют, что у них нет конфликта интересов.

\section{Список литературы}

[1] Askinazi L.G., Belokurov A.A., Gin D.B., Kornev V.A., Lebedev S.V., Shevelev A.E., Tukachinsky A.S., Zhubr N.A. // Nucl. Fusion. 2018. V. 58. N 8. P. 082003.

[2] Михайловский А.Б., Тимофеев А.В. // ЖЭТФ. 1963. Т. 44. B. 3. C. 919-921.

[3] Mikhailovsky A.B. // Nucl. Fusion. 1971. V. 11. N 4. P. 323-328.

[4] Pereverzev G.V., Yushmanov P.N. // Max-Plank IPP Report 5/98. Garching, Germany, 2002. $147 \mathrm{p}$. 